\title{
Maximum Likelihood Estimator of AUC for a Bi-Exponentiated Weibull Model
}

\author{
Fazhe Chang ${ }^{1}$ and Lianfen Qian ${ }^{1,2}$ \\ ${ }^{1}$ Department of Mathematical Sciences, Florida Atlantic University, Boca Raton, FL 33431, USA \\ ${ }^{2}$ College of Mathematics and Information Science, Wenzhou University, Zhejiang 325035, China \\ Correspondence should be addressed to Lianfen Qian; lqian@fau.edu
}

Received 29 April 2013; Accepted 25 July 2013

Academic Editors: N. Chernov, V. Makis, M. Montero, and O. Pons

Copyright (c) 2013 F. Chang and L. Qian. This is an open access article distributed under the Creative Commons Attribution License, which permits unrestricted use, distribution, and reproduction in any medium, provided the original work is properly cited.

For a Bi-Exponentiated Weibull model, the authors obtain a general AUC formula and derive the maximum likelihood estimator of AUC and its asymptotic property. A simulation study is carried out to illustrate the finite sample size performance.

\section{ROC Curve and AUC for a Bi-Exponentiated Weibull Model}

In medical science, a diagnostic test result called a biomarker $[1,2]$ is an indicator for disease status of patients. The accuracy of a medical diagnostic test is typically evaluated by sensitivity and specificity. Receiver Operating Characteristic (ROC) curve is a graphical representation of the relationship between sensitivity and specificity. The area under the ROC curve (AUC) is an overall performance measure for the biomarker. Hence the main issue in assessing the accuracy of a diagnostic test is to estimate the ROC curve and its AUC.

Suppose that there are two groups of study subjects: diseased and nondiseased. Let $T$ be a continuous biomarker. Assume that the larger $T$ is, the more likely a subject is diseased. That is, a subject is classified as positive or diseased status if $T>c$ and as negative or nondiseased status otherwise, where $c$ is a cutoff point. Let $D$ be the disease status: $D=1$ represents diseased population while $D=0$ represents nondiseased population. Sensitivity of $T$ is defined as the probability of being correctly classified as disease status and specificity as the probability of being correctly classified as nondisease status. That is,

$$
\begin{aligned}
& \text { sensitivity }(c)=P(T>c \mid D=1), \\
& \text { specificity }(c)=P(T<c \mid D=0) .
\end{aligned}
$$

Let $X=\{T \mid D=1\}$ and $Y=\{T \mid D=0\}$ be the biomarkers for diseased and nondiseased subjects with survival functions $S_{1}$ and $S_{0}$, respectively. Then

$$
\begin{gathered}
\operatorname{sensitivity}(c)=S_{1}(c), \\
\operatorname{specificity~}(c)=1-S_{0}(c) .
\end{gathered}
$$

The ROC function (curve) is defined as

$$
\operatorname{ROC}(t)=S_{1}\left(S_{0}^{-1}(t)\right), \quad \text { where } t=S_{0}(c) .
$$

Notice that ROC curve is a monotonic increasing curve in a unit square starting at $(0,0)$ and ending at $(1,1)$. A good biomarker has a very concave down ROC curve.

We say a bivariate random vector $(X, Y)$ is a $\mathrm{Bi}$-model if $X$ and $Y$ are independent and are from the same distribution family but with different parameters. Pepe [3] summarizes ROC analysis and notices that among Bi-model, Bi-normal model has been the most popular one. The ROC curve method traces back to Green and Swets [4] in the signal detection theory. Bi-normal model assumes the existence of a monotonic increasing transformation such that the biomarker can be transformed into normally distributed for both diseased and nondiseased patients. Pepe [3, Results 4.1 and 4.4] shows that the ROC curve of a biomarker is invariant under a monotone increasing transformation. Hence, a good estimator of ROC curve should satisfy this invariance property. 
The literature for ROC analysis under Bi-normal model is extensive and the majority of the results are for a continuous biomarker. For a continuous biomarker, Fushing and Turnbull [5] obtained the estimators and their asymptotic properties of the estimated ROC curve and AUC using the empirical based nonparametric and semiparametric approaches for $\mathrm{Bi}$ normal model. Metz et al. [6] studied properties of maximum likelihood estimator via discretization over finite support points. Zou and Hall [7] developed an MLE algorithm to estimate ROC curve under an unspecific or a specific monotonic transformation. Gu et al. [8] introduced a nonparametric method based on the Bayesian bootstrap technique to estimate ROC curve and compared the new method with several different semiparametric and nonparametric methods. Pepe and Cai [9] considered the ROC curve as the distribution of placement value and then estimated the ROC curve by maximizing the pseudolikelihood function of the estimated placement values. All the above methods require complicated computations. Zhou and Lin [10] proposed a semi-parametric maximum likelihood (ML) estimator of ROC which satisfies the property of invariance. Their method largely reduced the number of nuisance parameters and therefore the computational complexity.

In reality, the distribution of the biomarker may be skewed and the normality assumption is not reasonable. Under this situation, other models such as generalized exponential (GE), Weibull (WE), and gamma, to name a few, provide a reasonable alternative; see Faraggi et al. [11]. Another issue is that in the presence of two different biomarkers, the one with uniformly higher ROC curve is better. However, when two ROC curves cross, we cannot simply compare and find which one is better. Many different numerical indices have been proposed to summarize and compare ROC curves of different biomarkers [12-15]. The most popular summary index is AUC, defined as

$$
\mathrm{AUC}=\int_{0}^{1} \operatorname{ROC}(t) d t=P(X>Y)
$$

The estimation of AUC $=P(X>Y)$ is also of great importance in engineering reliability, typically, in stressstrength model. For example, if $X$ is the strength of a system which is subject to a stress $Y$, then $P(X>Y)$ is a measure of a system performance. The system fails if and only if at any time the applied stress is greater than its strength. During the past twenty years, many results have been obtained on the estimation of the probability under the different parametric models. Kotz et al. [16] give a comprehensive summarization about stress-strength model.

Many research results have been obtained for the estimation of AUC under popular survival models: Bi-exponential model [17], Bi-normal model [5, 18], Bi-gamma model [19], Bi-Burr type X [20-23], Bi-generalized exponential [24], BiWeibull model [25]. However, all the popular survival models such as generalized exponential (GE), Weibull (WE), and Gamma do not allow nonmonotonic failure rates which often occur in real practice. Furthermore, the common nonmonotonic failure rate in engineering and biological science involves bathtub shapes.
To overcome the aforementioned drawback, many models such as generalized gamma [26], generalized $F$ distribution [27], two families [28], a four-parameter family [29], and a three-parameter (IDB) family [30] have been proposed for modeling nonmonotone failure-rate data. Mudholkar and Srivasta [31] proposed an exponentiated Weibull (EW) model, an extension of both GE and WE models. Mudholkar and Hutson [32] studied some properties for the model. EW model not only includes distributions with unimodal and bathtub failure rates but also allows for a broader class of monotonic hazard rates. EW model may fit better than GE for some actual data. For instance, Khedhairi et al. [33] conclude that generalized Rayleigh model (a special case of EW model) is the best among the three models compared: exponential, generalized exponential, and generalized Rayleigh model.

In the research of estimation of $P(X>Y)$ under the different survival models, the most recent result in Baklizi [34] focuses the two-parameter Weibull model, which is a special case of our Bi-EW model. Baklizi assumes a common shape parameter and different scale parameter which is also studied in Kundu and Gupta [25]. In contrast to their studies, here we study the estimation of $P(X>Y)$ under the BiEW model which shares one shape parameter only. Since EW includes Weibull as a submodel, our results extend the ones in Baklizi [34] and Kundu and Gupta [25], as shown in Theorem 1. On the other hand, Baklizi [34] also studied Bayes estimator, which we have not studied. It will be a future project.

In this paper, we adopt AUC as a main summary index of ROC when comparing biomarkers under a Bi-Exponentiated Weibull (Bi-EW) model. We obtain a precise formula for the AUC and derive the MLE of AUC and its asymptotic distribution. The remaining of this paper is organized as follows. Section 2 introduces the Bi-EW model and obtains the theoretical AUC formula. Section 3 studies the maximum likelihood estimation of AUC under the Bi-EW model and derives the asymptotic normality of the estimated AUC. Section 4 reports the simulation results to compare the accuracy of the estimated parameters and estimated AUC for different sample sizes and various parameter settings in terms of absolute relative biases (ARB) and square root of mean square error (RMSE). The conclusion is given in Section 5 .

\section{A Bi-EW Model and Its AUC}

A random variable $X$ has an Exponentiated Weibull (EW) distribution if its distribution function is defined as

$$
F(x ; \alpha, \beta, \lambda)=\left(1-e^{-\lambda x^{\beta}}\right)^{\alpha}, \quad x>0, \alpha>0, \beta>0, \lambda>0 .
$$

Denote $X \sim \operatorname{EW}(\alpha, \beta, \lambda)$. Mudholkar and Srivasta [31] and Qian [35] show that this distribution allows both monotonic and bathtub shaped hazard rates. The latter shape is useful in reality. In this section, we first derive a general moment formula for the distribution and then obtain AUC formula for a Bi-EW model with a common shape parameter. 
2.1. General Moment Formula of Exponentiated Weibull. To fully understand a new family of distributions, it is important to derive its summary properties such as moment formulas.

Theorem 1. Let $X \sim E W(\alpha, \beta, \lambda)$. One has

$$
\begin{aligned}
E\left[X^{i \beta}(\ln X)^{j}\right] \\
=(-1)^{j} \frac{\alpha}{\lambda^{i} \beta^{j}} \sum_{l=0}^{j}\left(\begin{array}{l}
j \\
l
\end{array}\right) \Gamma^{(l)}(i+1) \\
\quad \times \sum_{k=0}^{\infty}(-1)^{k}\left(\begin{array}{c}
\alpha-1 \\
k
\end{array}\right) \frac{[\ln \lambda(k+1)]^{j-l}}{(k+1)^{i+1}},
\end{aligned}
$$

where $i, j=0,1,2,3, \ldots$ and $\Gamma(i)=\int_{0}^{\infty} e^{-t} t^{i-1} d t$

Proof. By the definition of the EW model, we have

$$
\begin{aligned}
& E\left[X^{i \beta}(\ln X)^{j}\right] \\
& =\alpha \lambda \beta \int_{0}^{\infty} x^{i \beta}(\ln x)^{j}\left(1-e^{-\lambda x^{\beta}}\right)^{\alpha-1} \\
& \quad \times e^{-\lambda x^{\beta}} x^{\beta-1} d x \\
& =\alpha \lambda \beta \sum_{k=0}^{\infty}(-1)^{k}\left(\begin{array}{c}
\alpha-1 \\
k
\end{array}\right) \\
& \quad \times \int_{0}^{\infty} x^{(i+1) \beta-1} e^{-\lambda(k+1) x^{\beta}}(\ln x)^{j} d x .
\end{aligned}
$$

By change of variables $t=\lambda(k+1) x^{\beta}$, we have

$$
d x=\frac{1}{\beta} \frac{t^{(1 / \beta)-1}}{[\lambda(k+1)]^{1 / \beta}} d t, \quad x^{(i+1) \beta-1}=\frac{t^{i+1-1 / \beta}}{[\lambda(k+1)]^{i+1-1 / \beta}} .
$$

Then the integral part in (7) is equal to

$$
\begin{aligned}
\int_{0}^{\infty} e^{-t}\left(\frac{\ln t-\ln \lambda(k+1)}{\beta}\right)^{j} \frac{1}{\beta} \frac{t^{i}}{[\lambda(k+1)]^{i+1}} d t \\
=\frac{1}{\beta^{j+1}[\lambda(k+1)]^{i+1}} \int_{0}^{\infty} e^{-t} t^{i}[\ln t-\ln \lambda(k+1)]^{j} d t \\
=\frac{1}{\beta^{j+1}[\lambda(k+1)]^{i+1}} \sum_{l=0}^{j}\left(\begin{array}{l}
j \\
l
\end{array}\right)[-\ln \lambda(k+1)]^{j-l} \\
\\
=\frac{\int_{0}^{\infty} e^{-t} t^{i}(\ln t)^{l} d t}{\beta^{j+1}[\lambda(k+1)]^{i+1}} \sum_{l=0}^{j}\left(\begin{array}{l}
j \\
l
\end{array}\right)[-\ln \lambda(k+1)]^{j-l} \Gamma^{(l)}(i+1) .
\end{aligned}
$$

Therefore, we have

$$
\begin{aligned}
& E\left[X^{i \beta}(\ln X)^{j}\right] \\
& =\alpha \lambda \beta \sum_{k=0}^{\infty}(-1)^{k}\left(\begin{array}{c}
\alpha-1 \\
k
\end{array}\right) \frac{1}{\beta^{j+1}[\lambda(k+1)]^{i+1}} \\
& \quad \times \sum_{l=0}^{j}\left(\begin{array}{l}
j \\
l
\end{array}\right)[-\ln \lambda(k+1)]^{j-l} \Gamma^{(l)}(i+1) \\
& =(-1)^{j} \frac{\alpha}{\lambda^{i} \beta^{j}} \sum_{l=0}^{j}(-1)^{l}\left(\begin{array}{l}
j \\
l
\end{array}\right) \Gamma^{(l)}(i+1) \\
& \quad \times \sum_{k=0}^{\infty}\left(\begin{array}{c}
\alpha-1 \\
k
\end{array}\right) \frac{[\ln \lambda(k+1)]^{j-l}}{(k+1)^{i+1}} .
\end{aligned}
$$

Remark 2. Theorem 1 covers the following results as special cases.

Case 1. If $j=0$, and $i \beta=k$, then it reduces to the formula in Choudlhury [36], the simple moments of EW family. One has

$$
\begin{aligned}
\mu_{k}= & E\left(X^{k}\right)=\alpha \lambda^{-k / \beta} \Gamma\left(\frac{k}{\beta}+1\right) \\
& \times \sum_{i=0}^{\alpha-1}(-1)^{i}\left(\begin{array}{c}
\alpha-1 \\
i
\end{array}\right) \frac{1}{(i+1)^{k /(\beta+1)}} .
\end{aligned}
$$

Moreover, if $k \beta^{-1}=r$ is positive integer, then

$$
\mu_{k}=\left.\alpha(-\lambda)^{-r}\left[\frac{d^{r}}{d s^{r}} B(s, \alpha)\right]\right|_{s=1}
$$

where $B(s, \alpha)=\int_{0}^{1} x^{s-1}(1-x)^{\alpha-1} d x$.

Case 2. If $j=0$, and $\beta=1$, it reducces to the moments of Generalized exponential family in Kunda and Gupta [24].

Case 3. If $j=0, \alpha=1$, and $i \beta=n$, it reduces to the moments of Weibull family in Kunda and Gupta [25].

2.2. AUC under a Bi-EW Model. In this subsection, we derive the general formula of AUC under a Bi-EW model with a common shape parameter. That is, we assume that $X$ and $Y$ are independent and with the following EW distribution functions:

$$
\begin{array}{r}
F_{1}\left(x ; \alpha_{1}, \beta, \lambda_{1}\right)=\left(1-e^{-\lambda_{1} x^{\beta}}\right)^{\alpha_{1}}, \quad x>0, \alpha_{1}>0, \quad \beta>0, \\
\lambda_{1}>0, \\
F_{0}\left(y ; \alpha, \beta, \lambda_{2}\right)=\left(1-e^{-\lambda_{2} y^{\beta}}\right)^{\alpha_{2}}, \quad y>0, \alpha_{2}>0, \quad \beta>0, \\
\lambda_{2}>0,
\end{array}
$$


where the two distribution functions share a common shape parameter $\beta$.

Theorem 3. Suppose that $(X, Y)$ follows the Bi-EW model with the above distribution functions. Then

$$
A U C=\sum_{i=0}^{\infty} \sum_{j=1}^{\infty}\left(\begin{array}{c}
\alpha_{2} \\
i
\end{array}\right)\left(\begin{array}{c}
\alpha_{1} \\
j
\end{array}\right)(-1)^{i+j-1} \frac{\lambda_{1} j}{\lambda_{1} j+\lambda_{2} i} .
$$

Proof. By Taylor expansion, we have

$$
\begin{aligned}
& \left(1-e^{-\lambda_{2} x^{\beta}}\right)^{\alpha_{2}}=\sum_{i=0}^{\infty}\left(\begin{array}{c}
\alpha_{2} \\
i
\end{array}\right)\left(-e^{-\lambda_{2} x^{\beta}}\right)^{i}, \\
& \left(1-e^{-\lambda_{1} x^{\beta}}\right)^{\alpha_{1}}=\sum_{j=0}^{\infty}\left(\begin{array}{c}
\alpha_{1} \\
j
\end{array}\right)\left(-e^{-\lambda_{1} x^{\beta}}\right)^{j} .
\end{aligned}
$$

Thus,

$$
\begin{aligned}
A U C= & \int_{0}^{\infty} F_{0}\left(x ; \alpha_{2}, \beta, \lambda_{2}\right) d F_{1}\left(x ; \alpha_{1}, \beta, \lambda_{1}\right) \\
= & \int_{0}^{\infty} \sum_{i=0}^{\infty}\left(\begin{array}{c}
\alpha_{2} \\
i
\end{array}\right)\left(-e^{-\lambda_{2} x^{\beta}}\right)^{i} \\
& \times \sum_{j=1}^{\infty}\left(\begin{array}{c}
\alpha_{1} \\
j
\end{array}\right) j\left(-e^{-\lambda_{1} x^{\beta}}\right)^{j-1} e^{-\lambda_{1} x^{\beta}} \lambda_{1} \beta x^{\beta-1} d x \\
= & \sum_{i=0}^{\infty} \sum_{j=1}^{\infty} \int_{0}^{\infty}\left(\begin{array}{c}
\alpha_{2} \\
i
\end{array}\right)\left(\begin{array}{c}
\alpha_{1} \\
j
\end{array}\right) j(-1)^{i+j-1} \lambda_{1} e^{-\left(\lambda_{2} i+\lambda_{1} j\right) x^{\beta}} d x^{\beta} \\
= & \sum_{i=0}^{\infty} \sum_{j=1}^{\infty}\left(\begin{array}{c}
\alpha_{2} \\
i
\end{array}\right)\left(\begin{array}{c}
\alpha_{1} \\
j
\end{array}\right)(-1)^{i+j-1} \lambda_{1} j \int_{0}^{\infty} e^{-\left(\lambda_{2} i+\lambda_{1} j\right) x^{\beta}} d x^{\beta} \\
= & \sum_{i=0}^{\infty} \sum_{j=1}^{\infty}\left(\begin{array}{c}
\alpha_{2} \\
i
\end{array}\right)\left(\begin{array}{c}
\alpha_{1} \\
j
\end{array}\right)(-1)^{i+j-1} \frac{\lambda_{1} j}{\lambda_{1} j+\lambda_{2} i} .
\end{aligned}
$$

Remark 4. (i) Note that the exact expression of AUC is independent of the common parameter $\beta$. This is similar to the cases in Bi-WE and Bi-GE models; see Kundu and Gupta $[24,25]$.

(ii) When $\alpha_{1}=\alpha_{2}=1$, (14) reduces to the AUC for a Bi-GE model.

(iii) When $\lambda_{1}=\lambda_{2}$, (14) reduces to the AUC for a Bi-WE model. To see (iii), we actually prove the following corollary.

Corollary 5. For $\alpha_{1}>0$ and $\alpha_{2}>0$, one has

$$
\sum_{i=0}^{\infty} \sum_{j=1}^{\infty}\left(\begin{array}{c}
\alpha_{2} \\
i
\end{array}\right)\left(\begin{array}{c}
\alpha_{1} \\
j
\end{array}\right)(-1)^{i+j-1} \frac{j}{j+i}=\frac{\alpha_{1}}{\alpha_{1}+\alpha_{2}} .
$$

Proof. Denote

$$
g(s)=\frac{\partial}{\partial s} \int_{0}^{1} \sum_{i=0}^{\infty} \sum_{j=1}^{\infty}\left(\begin{array}{c}
\alpha_{2} \\
i
\end{array}\right)\left(\begin{array}{c}
\alpha_{1} \\
j
\end{array}\right)(-1)^{i+j-1} t^{i+j-1} s^{j} d t .
$$

Then, on one hand we have

$$
\begin{aligned}
g(s) & =\frac{\partial}{\partial s} \int_{0}^{1} \sum_{i=0}^{\infty}\left(\begin{array}{c}
\alpha_{2} \\
i
\end{array}\right)(-1)^{i} t^{i-1} \sum_{j=1}^{\infty}\left(\begin{array}{c}
\alpha_{1} \\
j
\end{array}\right)(-1)^{j-1}(t s)^{j} d t \\
& =\frac{\partial}{\partial s} \int_{0}^{1} \frac{1}{t(1-t)^{\alpha_{2}}\left(1-(1-t s)^{\alpha_{1}}\right)} d t \\
& =\int_{0}^{1} \frac{1}{t}(1-t)^{\alpha_{2}}(1-t s)^{\alpha_{1}-1} \alpha_{1} t d t \\
& =\alpha_{1} \int_{0}^{1}(1-t)^{\alpha_{2}}(1-t s)^{\alpha_{1}-1} d t .
\end{aligned}
$$

On the other hand, we have

$$
\begin{aligned}
g(s) & =\frac{\partial}{\partial s} \sum_{i=0}^{\infty} \sum_{j=1}^{\infty}\left(\begin{array}{c}
\alpha_{2} \\
i
\end{array}\right)\left(\begin{array}{c}
\alpha_{1} \\
j
\end{array}\right)(-1)^{i+j-1} s^{j} \frac{t^{i+j}}{i+j} \\
& =\sum_{i=0}^{\infty} \sum_{j=1}^{\infty}\left(\begin{array}{c}
\alpha_{2} \\
i
\end{array}\right)\left(\begin{array}{c}
\alpha_{1} \\
j
\end{array}\right)(-1)^{i+j-1} \frac{j}{i+j} s^{j-1} t^{i+j} .
\end{aligned}
$$

Let $s=1$; then (19) implies that

$$
g(1)=\frac{\alpha_{1}}{\alpha_{1}+\alpha_{2}}
$$

while (20) implies that

$$
g(1)=\sum_{i=0}^{\infty} \sum_{j=1}^{\infty}\left(\begin{array}{c}
\alpha_{2} \\
i
\end{array}\right)\left(\begin{array}{c}
\alpha_{1} \\
j
\end{array}\right)(-1)^{i+j-1} \frac{j}{i+j} .
$$

This completes the proof of the corollary.

\section{Maximum Likelihood Estimator of AUC and Its Asymptotic Property under the Bi-EW Model}

To estimate AUC under the Bi-EW model, we adopt the plug in method. That is, we first obtain the maximum likelihood estimator of the model parameters and then plug it into (14) to get the MLE of AUC.

3.1. Maximum Likelihood Estimator of AUC under the Bi-EW Model. Let $X_{1}, X_{2}, \ldots, X_{m}$ and $Y_{1}, Y_{2}, \ldots, Y_{n}$ be independent random samples from $X \sim F_{1}$ and $Y \sim F_{0}$, respectively. 
Denote the model parameter vector by $\theta=\left(\theta_{1}, \ldots, \theta_{5}\right)=$ $\left(\alpha_{1}, \alpha_{2}, \beta, \lambda_{1}, \lambda_{2}\right)$. Then the log-likelihood function is

$$
\begin{aligned}
l(\theta)= & m \ln \alpha_{1}+m \ln \lambda_{1}+m \ln \beta+n \ln \alpha_{2}+n \ln \lambda_{2} \\
& +n \ln \beta+\left(\alpha_{1}-1\right) \sum_{i=1}^{m} \ln \left(1-e^{-\lambda_{1} x_{i}^{\beta}}\right) \\
& +\left(\alpha_{2}-1\right) \sum_{j=1}^{n} \ln \left(1-e^{-\lambda_{2} y_{i}^{\beta}}\right) \\
& -\sum_{i=1}^{m} \lambda_{1} x_{i}^{\beta}+(\beta-1) \sum_{i=1}^{m} \ln x_{i} \\
& -\sum_{j=1}^{n} \lambda_{2} y_{j}^{\beta}+(\beta-1) \sum_{j=1}^{n} \ln y_{i} .
\end{aligned}
$$

We take derivatives with respect to the parameters to get the following score equations:

$$
\begin{gathered}
\frac{\partial l}{\partial \alpha_{1}}=\frac{m}{\alpha_{1}}+\sum_{i=1}^{m} \ln \left(1-e^{-\lambda_{1} x_{i}^{\beta}}\right)=0, \\
\frac{\partial l}{\partial \alpha_{2}}=\frac{n}{\alpha_{2}}+\sum_{j=1}^{n} \ln \left(1-e^{-\lambda_{2} y_{j}^{\beta}}\right)=0, \\
\frac{\partial l}{\partial \lambda_{1}}=\frac{m}{\lambda_{1}}-\sum_{i=1}^{m} x_{i}^{\beta}+\left(\alpha_{1}-1\right) \sum_{i=1}^{m} \frac{e^{-\lambda_{1} x_{i}^{\beta}} x_{i}^{\beta}}{1-e^{-\lambda_{1} x_{i}^{\beta}}}=0, \\
\frac{\partial l}{\partial \lambda_{2}}=\frac{n}{\lambda_{2}}-\sum_{j=1}^{n} y_{j}^{\beta}+\left(\alpha_{2}-1\right) \sum_{j=1}^{n} \frac{e^{-\lambda_{2} y_{j}^{\beta}} y_{j}^{\beta}}{1-e^{-\lambda_{2} y_{j}^{\beta}}}=0, \\
\frac{\partial l}{\partial \beta}=\frac{m+n}{\beta}-\lambda_{1} \sum_{i=1}^{m} x_{i}^{\beta} \ln x_{i}-\lambda_{2} \sum_{j=1}^{n} y_{j}^{\beta} \ln y_{j} \\
+\sum_{i=1}^{m} \ln x_{i}+\sum_{j=1}^{n} \ln y_{j} \\
+\left(\alpha_{1}-1\right) \sum_{i=1}^{m} \frac{e^{-\lambda_{1} x_{i}^{\beta}} \lambda_{1} x_{i}^{\beta} \ln x_{i}}{1-e^{-\lambda_{1} x_{i}^{\beta}}} \\
+\left(\alpha_{2}-1\right) \sum_{j=1}^{n} \frac{e^{-\lambda_{2} y_{j}^{\beta}} \lambda_{2} y_{j}^{\beta} \ln y_{j}}{1-e^{-\lambda_{2} y_{j}^{\beta}}}=0 .
\end{gathered}
$$

The MLE $\widehat{\theta}$ of $\theta$ is the numerical solution of the above score equations. Plugging $\widehat{\theta}$ into (14), we obtain the maximum likelihood estimator of AUC as below:

$$
\widehat{\mathrm{AUC}}=\sum_{i=0}^{\infty} \sum_{j=1}^{\infty}\left(\begin{array}{c}
\widehat{\alpha}_{2} \\
i
\end{array}\right)\left(\begin{array}{c}
\widehat{\alpha}_{1} \\
j
\end{array}\right)(-1)^{i+j-1} \frac{\widehat{\lambda}_{1} j}{\widehat{\lambda}_{1} j+\widehat{\lambda}_{2} i} .
$$

3.2. Asymptotic Normality of the Estimated AUC. In this section, we obtain the asymptotic distribution of $\widehat{\theta}=\left(\widehat{\alpha}_{1}, \widehat{\alpha}_{2}\right.$, $\widehat{\beta}, \widehat{\lambda}_{1}, \widehat{\lambda}_{2}$ ) and hence, by the continuous mapping theorem, the asymptotic distribution of the estimated AUC. We need to introduce some notation. Let

$$
\mu_{j, k}^{X}=E\left(X^{\beta(j+1)}(\ln X)^{k}\right),
$$

$\mu_{j, k}^{Y}=E\left(Y^{\beta(j+1)}(\ln Y)^{k}\right), \quad$ for $j, k=0,1,2, \ldots$,

$$
\mathbf{A}(\theta)=\left(\begin{array}{ccccc}
a_{11} & 0 & a_{13} & a_{14} & 0 \\
0 & a_{22} & a_{23} & 0 & a_{25} \\
a_{31} & a_{32} & a_{33} & a_{34} & a_{35} \\
a_{41} & 0 & a_{43} & a_{44} & 0 \\
0 & a_{52} & a_{53} & 0 & a_{55}
\end{array}\right) .
$$

Then

$$
\begin{aligned}
& a_{11}=\frac{1}{\alpha_{1}^{2}}, \quad a_{22}=\frac{1}{\alpha_{2}^{2}}, \\
& a_{13}=a_{31}=-\lambda_{1} \sum_{i=0}^{\infty} \sum_{j=0}^{\infty} \frac{\left(-\lambda_{1}(1+i)\right)^{j}}{j !} \mu_{j, 1}^{Y},
\end{aligned}
$$$$
a_{23}=a_{32}=-\frac{1}{\sqrt{p}} \lambda_{2} \sum_{i=0}^{\infty} \sum_{j=0}^{\infty} \frac{\left(-\lambda_{2}(1+i)\right)^{j}}{j !} \mu_{j, 1}^{Y} \text {, }
$$$$
a_{25}=a_{52}=-\sum_{i=0}^{\infty} \sum_{j=0}^{\infty} \frac{\left(-\lambda_{2}(1+i)\right)^{j}}{j !} \mu_{j, 0}^{Y},
$$$$
a_{33}=\lambda_{1} \mu_{0,2}^{X}+\frac{1}{\sqrt{p}} \lambda_{2} \mu_{0,2}^{Y}+\frac{1+p}{\sqrt{p} \beta^{2}}
$$$$
-\lambda_{1}\left(\alpha_{1}-1\right)
$$$$
\times \sum_{i=0}^{\infty} \sum_{j=0}^{\infty} \frac{\left(1-\lambda_{1}(1+i)\right)}{j !}\left(-\lambda_{1}(1+i)\right)^{j} \mu_{j, 2}^{X}
$$$$
-\frac{1}{\sqrt{p}} \lambda_{2}\left(\alpha_{2}-1\right)
$$

$$
\times \sum_{i=0}^{\infty} \sum_{j=0}^{\infty} \frac{\left(-\lambda_{1}(1+i)\right)}{j !}\left(1-\lambda_{2}(1+i)\right)^{j} \mu_{j, 2}^{Y},
$$

$$
a_{34}=a_{43}=\mu_{0,1}^{X}-\left(\alpha_{1}-1\right)
$$$$
\times \sum_{i=0}^{\infty} \sum_{j=0}^{\infty} \frac{\left(1-\lambda_{1}(1+i)\right)}{j !}\left(-\lambda_{1}(1+i)\right)^{j} \mu_{j, 1}^{X},
$$$$
a_{35}=a_{53}=\frac{1}{\sqrt{p}} \mu_{0,1}^{Y}-\frac{1}{\sqrt{p}}\left(\alpha_{2}-1\right)
$$

$$
\times \sum_{i=0}^{\infty} \sum_{j=0}^{\infty} \frac{\left(1-\lambda_{2}(1+i)\right)}{j !}\left(-\lambda_{2}(1+i)\right)^{j} \mu_{j, 1}^{Y} \text {, }
$$

$a_{44}=\frac{1}{\lambda_{1}^{2}}+\left(\alpha_{1}-1\right) \sum_{i=0}^{\infty} \sum_{j=0}^{\infty} \frac{(i+1)^{j+1}}{j !}\left(-\lambda_{1}\right)^{j} \mu_{j+1,0}^{X}$,

$a_{55}=\frac{1}{\lambda_{2}^{2}}+\left(\alpha_{2}-1\right) \sum_{i=0}^{\infty} \sum_{j=0}^{\infty} \frac{(i+1)^{j+1}}{j !}\left(-\lambda_{2}\right)^{j} \mu_{j+1,0}^{Y}$. 


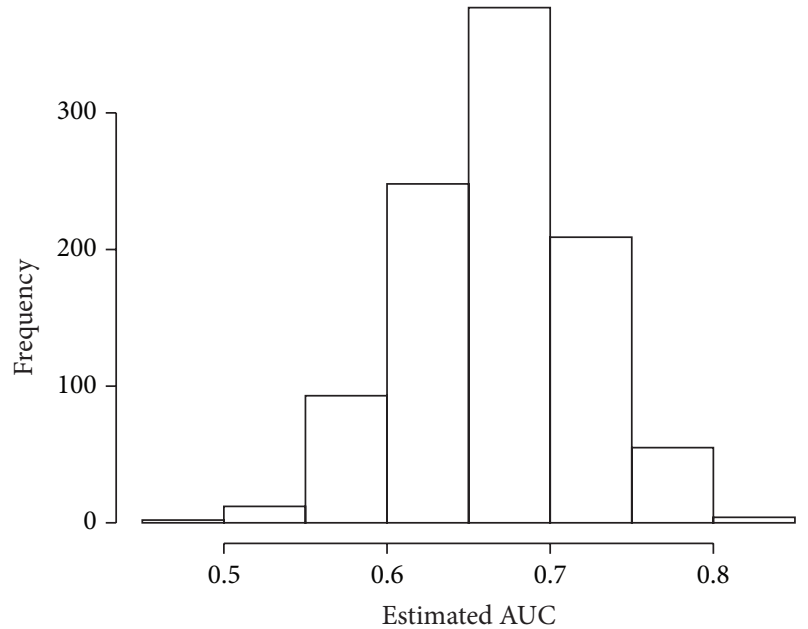

(a)

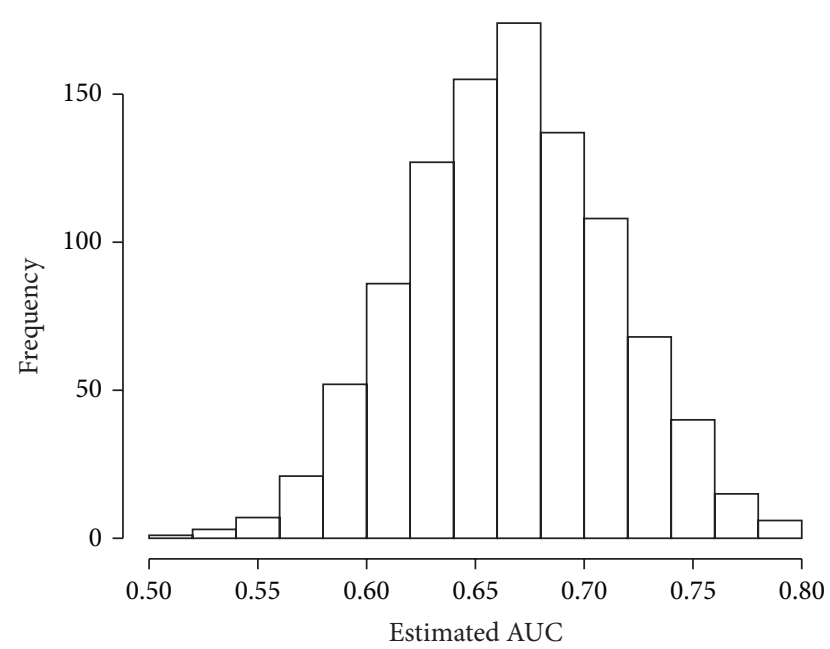

(c)

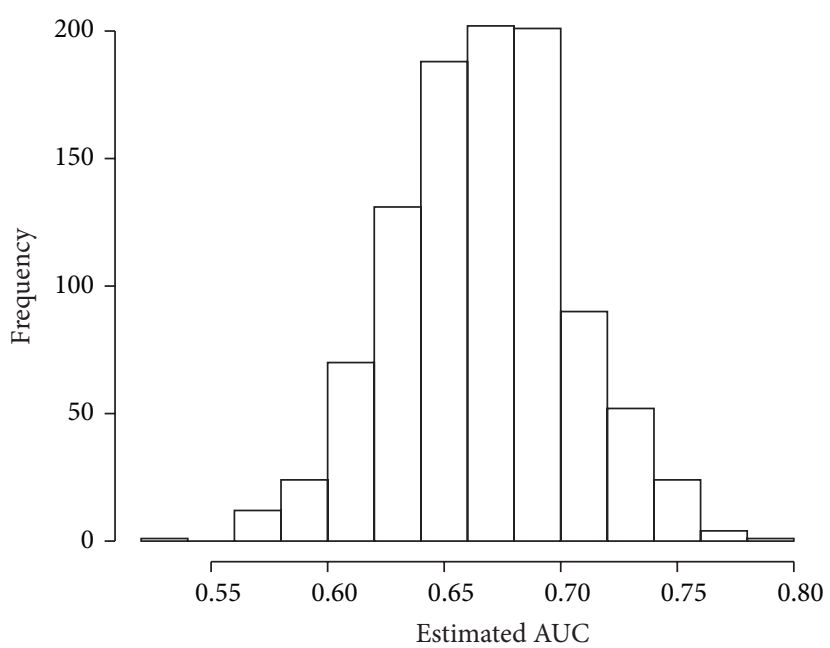

(b)

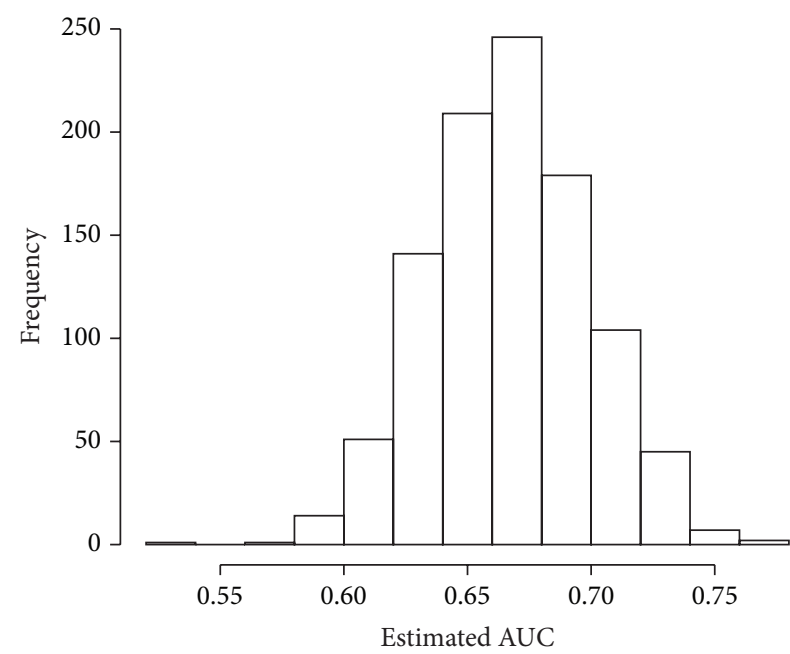

(d)

Figure 1: Histograms of the estimated AUC for $(\mathrm{a})(m, n)=(50,50),(\mathrm{b})(m, n)=(100,100),(\mathrm{c})(m, n)=(50,100)$, and $(\mathrm{d})(m, n)=(100,200)$.

We denote $D=\operatorname{diag}(\sqrt{m}, \sqrt{n}, \sqrt{m}, \sqrt{m}, \sqrt{n})$. Notice that EW family satisfies all the regularity conditions almost everywhere. Now we are ready to state the following theorem.

Theorem 6. Suppose $(X, Y)$ follows the Bi-EW model with the model parameter $\theta$. If $m \rightarrow \infty$ and $n \rightarrow \infty$, but $m / n \rightarrow p$, for $0<p<\infty$, then one has the following.

(a) The MLE $\hat{\theta}$ of $\theta$ is asympotic normal:

$$
D(\widehat{\theta}-\theta) \longrightarrow N_{5}\left(0, \mathbf{A}^{-1}(\theta)\right)
$$

(b) Part (a), the continuous mapping theorem, and Delta method imply that

$$
\sqrt{m}(\widehat{A U C}-A U C) \longrightarrow N(0, B),
$$

where

$$
\begin{gathered}
B=\left(\frac{\partial A U C}{\partial \theta}\right) \mathbf{A}^{-1}(\theta)\left(\frac{\partial A U C}{\partial \theta}\right)^{T}, \\
\frac{\partial A U C}{\partial \theta}=\left(\frac{\partial A U C}{\partial \alpha_{1}}, \frac{\partial A U C}{\partial \alpha_{2}}, 0, \frac{\partial A U C}{\partial \beta_{1}}, \frac{\partial A U C}{\partial \beta_{2}}\right) .
\end{gathered}
$$

\section{A Simulation Study}

In this section we present some results based on Monte Carlo simulation to check the performance of the MLE of parameters and AUC. We consider small to moderate sample sizes: $m, n=50,100$, and 200 . We set two scale parameters: $\lambda_{1}=\lambda_{2}=1$. The common shape parameter is $\beta=2$. The other shape parameters are $\alpha_{1}=2$ and $\alpha_{2}=1,2,3,4$, respectively. The simulation is based on 1000 replicates.

Figure 1 illustrates the histograms of the estimated AUC under the sample sizes $(m, n)=(50,50),(100,100),(50,100)$, $(100,200)$ when the true parameters are $\alpha_{1}=2, \alpha_{2}=4$, 
TABLE 1: ARB and RMSE of five estimated parameters $\left(\widehat{\alpha}_{1}, \widehat{\alpha}_{2}, \widehat{\beta}, \widehat{\lambda}_{1}\right.$, and $\left.\widehat{\lambda}_{2}\right)$ and $\widehat{\mathrm{AUC}}$, when $\alpha_{1}=2, \beta=2, \lambda_{1}=1$, and $\lambda_{2}=1$ and with different values of $\alpha_{2}$.

\begin{tabular}{|c|c|c|c|c|c|}
\hline$(m, n)$ & MLE & $\alpha_{2}=1$ & $\alpha_{2}=2$ & $\alpha_{2}=3$ & $\alpha_{2}=4$ \\
\hline \multirow{6}{*}{$(50,50)$} & $\widehat{\alpha}_{1}$ & $0.3721(1.6569)$ & $0.35630(2.211)$ & 0.3607 (1.5814) & $0.3995(1.950)$ \\
\hline & $\widehat{\alpha}_{2}$ & $0.2556(1.1068)$ & $0.3645(1.2601)$ & $0.4204(1.5814)$ & $0.5547(2.1276)$ \\
\hline & $\widehat{\beta}$ & $0.0801(0.7525)$ & $0.0675(0.6655)$ & $0.0615(0.6679)$ & $0.0525(0.6243)$ \\
\hline & $\hat{\lambda}_{1}$ & $0.0842(0.5661)$ & $0.0756(0.5421)$ & $0.961(0.5442)$ & $0.0979(0.5683)$ \\
\hline & $\widehat{\lambda}_{2}$ & $0.0913(0.5235)$ & $0.0805(0.5493)$ & $0.0916(0.560)$ & $0.119(0.6189)$ \\
\hline & $\widehat{\mathrm{AUC}}$ & $0.0309(0.0648)$ & $0.0187(0.0734)$ & $0.0074(0.08)$ & $0.0078(0.0648)$ \\
\hline \multirow{6}{*}{$(50,100)$} & $\widehat{\alpha}_{1}$ & $0.1872(1.6205)$ & $0.3035(2.1506)$ & $0.2333(1.5885)$ & $0.2205(1.9536)$ \\
\hline & $\widehat{\alpha}_{2}$ & $0.0823(0.4995)$ & $0.2404(1.8192)$ & $0.2507(2.6231)$ & $0.2896(2.1170)$ \\
\hline & $\widehat{\beta}$ & $0.0636(0.5464)$ & $0.0293(0.5054)$ & $0.0271(0.4935)$ & $0.0467(0.5228)$ \\
\hline & $\widehat{\lambda}_{1}$ & $0.0348(0.4380)$ & $0.0985(0.4777)$ & $0.0859(0.4421)$ & $0.0653(0.4614)$ \\
\hline & $\hat{\lambda}_{2}$ & $0.0217(0.3694)$ & $0.0751(0.4532)$ & $0.0762(0.4510)$ & $0.0612(0.4861)$ \\
\hline & $\widehat{\mathrm{AUC}}$ & $0.0072(0.060)$ & $0.005(0.0591)$ & $0.0037(0.0565)$ & $0.0058(0.0424)$ \\
\hline \multirow{6}{*}{$(100,50)$} & $\widehat{\alpha}_{1}$ & $0.1609(1.3638)$ & $0.1689(1.5535)$ & $0.1774(1.3754)$ & 0.1731 (1.4751) \\
\hline & $\widehat{\alpha}_{2}$ & 0.1379 (0.5709) & $0.2031(1.6370)$ & $0.2773(1.9342)$ & $0.3209(1.7746)$ \\
\hline & $\widehat{\beta}$ & $0.0467(0.5281)$ & $0.0455(0.4998)$ & $0.0320(0.4814)$ & $0.0425(0.5079)$ \\
\hline & $\hat{\lambda}_{1}$ & $0.0399(0.4085)$ & $0.0430(0.4129)$ & $0.0602(0.3962)$ & $0.0461(0.4191)$ \\
\hline & $\widehat{\lambda}_{2}$ & $0.0709(0.4078)$ & $0.0524(0.4300)$ & $0.0824(0.4498)$ & $0.0668(0.4889)$ \\
\hline & $\widehat{\mathrm{AUC}}$ & $0.0048(0.0519)$ & $0.0031(0.0489)$ & $0.0017(0.0469)$ & $0.0056(0.0435)$ \\
\hline \multirow{6}{*}{$(100,100)$} & $\widehat{\alpha}_{1}$ & $0.1205(1.1180)$ & $0.1322(1.2528)$ & $0.1396(1.2052)$ & 0.1276 (1.1109) \\
\hline & $\widehat{\alpha}_{2}$ & $0.0726(0.4195)$ & $0.1291(1.2499)$ & $0.1842(2.1241)$ & $0.1295(2.0528)$ \\
\hline & $\widehat{\beta}$ & $0.0367(0.4324)$ & $0.0346(0.4317)$ & $0.0290(0.4219)$ & $0.0323(0.4281)$ \\
\hline & $\widehat{\lambda}_{1}$ & $0.0318(0.3521)$ & $0.0354(0.3687)$ & $0.0469(0.3720)$ & $0.0369(0.3644)$ \\
\hline & $\widehat{\lambda}_{2}$ & $0.029(0.3286)$ & $0.0323(0.3739)$ & $0.0593(0.3962)$ & $0.0452(0.3954)$ \\
\hline & $\widehat{\mathrm{AUC}}$ & $0.0036(0.0412)$ & $0.003(0.0458)$ & $0.0018(0.0331)$ & $0.0031(0.040)$ \\
\hline \multirow{6}{*}{$(200,200)$} & $\widehat{\alpha}_{1}$ & $0.0668(0.6871)$ & $0.0569(0.6666)$ & $0.0700(0.6368)$ & $0.0602(0.6553)$ \\
\hline & $\widehat{\alpha}_{2}$ & $0.0404(0.2707)$ & $0.0637(0.6581)$ & 0.0833 (1.1019) & $0.0965(0.7324)$ \\
\hline & $\widehat{\beta}$ & $0.0152(0.2879)$ & $0.0135(0.2853)$ & $0.0090(0.2791)$ & $0.0102(0.2756)$ \\
\hline & $\widehat{\lambda}_{1}$ & $0.0214(0.2487)$ & $0.0215(0.2457)$ & $0.0324(0.2399)$ & $0.0217(0.2430)$ \\
\hline & $\widehat{\lambda}_{2}$ & $0.0162(0.2274)$ & $0.0222(0.2419)$ & $0.0292(0.2562)$ & $0.0280(0.2715)$ \\
\hline & $\widehat{\mathrm{AUC}}$ & $0.0021(0.0256)$ & $0.0033(0.0267)$ & $0.0031(0.0270)$ & $0.0013(0.0264)$ \\
\hline
\end{tabular}

$\beta=2$, and $\lambda_{1}=\lambda_{2}=1$. Obviously, as the sample size increases, the histogram tends to be more symmetric bell shaped. Therefore, the MLE of AUC performs well for the moderate sample size.

We report the absolute relative biases (ARB) and the square root of mean squared errors (RMSEs) for the estimators of $\theta=\left(\theta_{1}, \ldots, \theta_{5}\right)$ and AUC defined as below:

$$
\begin{gathered}
\operatorname{ARB}\left(\widehat{\theta}_{j}\right)=\frac{1}{1000} \sum_{i=1}^{1000}\left|\frac{\widehat{\theta}_{j}^{i}-\theta_{j}}{\theta_{j}}\right|, \\
\operatorname{RMSE}\left(\widehat{\theta}_{j}\right)=\sqrt{\frac{1}{1000} \sum_{i=1}^{1000}\left(\widehat{\theta}_{j}^{i}-\theta_{j}\right)^{2}, \quad j=1,2,3,4,5,}
\end{gathered}
$$

where $\widehat{\theta}_{j}^{i}$ is the estimates of $\theta_{j}$ for the $i$ th replicate.

Recall that

$$
\mathrm{AUC}=\int_{0}^{\infty} F_{0}\left(x ; \alpha_{2}, \beta, \lambda_{2}\right) d F_{1}\left(x ; \alpha_{1}, \beta, \lambda_{1}\right) .
$$

The estimator $\widehat{\mathrm{AUC}}$ and true value AUC can be computed by Riemann sums

$$
\begin{gathered}
\sum_{x=0.0001}^{b} F_{0}\left(x ; \widehat{\alpha}_{2}, \widehat{\beta}, \hat{\lambda}_{2}\right) f_{1}\left(x ; \widehat{\alpha}_{1}, \widehat{\beta}, \widehat{\lambda}_{1}\right) \Delta x, \\
\sum_{x=0.0001}^{b} F_{0}\left(x ; \alpha_{2}, \beta, \lambda_{2}\right) f_{1}\left(x ; \alpha_{1}, \beta, \lambda_{1}\right) \Delta x,
\end{gathered}
$$

respectively. Here, we choose $b=4$ and $\Delta x=0.0001$. The interval $[0,4]$ is divided evenly with each subinterval length 0.0001 and $x$ takes the value of the right end of each subinterval. We have verified that the summation keeps stable for any larger value $b$ and smaller value $\Delta x$.

Similarly,

$$
\operatorname{ARB}(\widehat{\mathrm{AUC}})=\frac{1}{1000} \sum_{i=1}^{1000}\left|\frac{\widehat{\mathrm{AUC}}_{i}-\mathrm{AUC}}{\mathrm{AUC}}\right|,
$$


TABLE 2: ARB and RMSE of five estimated parameters $\left(\widehat{\alpha}_{1}, \widehat{\alpha}_{2}, \widehat{\beta}, \widehat{\lambda}_{1}\right.$, and $\left.\widehat{\lambda}_{2}\right)$ and $\widehat{\mathrm{AUC}}$, when $\alpha_{1}=2, \alpha_{2}=3, \lambda_{1}=1$, and $\lambda_{2}=1$ and with different values of $\beta$.

\begin{tabular}{|c|c|c|c|c|}
\hline$(m, n)$ & MLE & $\beta=2$ & $\beta=2.5$ & $\beta=3$ \\
\hline \multirow{6}{*}{$(50,50)$} & $\widehat{\alpha}_{1}$ & $0.1925(1.7453)$ & $0.1646(1.7159)$ & $0.1863(1.7626)$ \\
\hline & $\widehat{\alpha}_{2}$ & $0.4685(2.245)$ & $0.3917(1.9021)$ & $0.4641(2.348)$ \\
\hline & $\widehat{\beta}$ & $0.0554(0.6402)$ & $0.0912(0.7801)$ & $0.0558(0.9117)$ \\
\hline & $\hat{\lambda}_{1}$ & $0.1044(0.5624)$ & $0.0700(0.5442)$ & $0.0800(0.5353)$ \\
\hline & $\widehat{\lambda}_{2}$ & $0.1048(0.5779)$ & $0.0746(0.5624)$ & $0.0939(0.5719)$ \\
\hline & $\widehat{\mathrm{AUC}}$ & $0.0067(0.0556)$ & $0.0026(0.0557)$ & $0.0036(0.0529)$ \\
\hline \multirow{6}{*}{$(50,80)$} & $\widehat{\alpha}_{1}$ & $0.2538(1.8319)$ & $0.2472(2.0245)$ & $0.2775(2.0910)$ \\
\hline & $\widehat{\alpha}_{2}$ & $0.2818(2.0819)$ & $0.2691(2.245)$ & $0.3046(2.3255)$ \\
\hline & $\hat{\beta}$ & $0.0401(0.5415)$ & $0.0557(0.6907)$ & $0.0475(0.8629)$ \\
\hline & $\hat{\lambda}_{1}$ & $0.0825(0.4706)$ & $0.0635(0.4821)$ & $0.0815(0.4803)$ \\
\hline & $\widehat{\lambda}_{2}$ & $0.0724(0.4790)$ & $0.0489(0.4931)$ & $0.0741(0.4952)$ \\
\hline & $\widehat{\mathrm{AUC}}$ & $0.0045(0.0490)$ & $0.0015(0.0510)$ & $0.0008(0.050)$ \\
\hline \multirow{6}{*}{$(80,50)$} & $\widehat{\alpha}_{1}$ & $0.1846(1.6341)$ & $0.2311(1.7011)$ & $0.2874(1.7425)$ \\
\hline & $\widehat{\alpha}_{2}$ & $0.2515(2.2201)$ & $0.3733(2.2412)$ & $0.3969(1.956)$ \\
\hline & $\widehat{\beta}$ & $0.0501(0.4921)$ & $0.0414(0.701)$ & $0.0339(0.8101)$ \\
\hline & $\hat{\lambda}_{1}$ & $0.0380(0.4365)$ & $0.0665(0.4577)$ & $0.0905(0.4761)$ \\
\hline & $\hat{\lambda}_{2}$ & $0.0443(0.4681)$ & $0.0952(0.5278)$ & $0.0962(0.5221)$ \\
\hline & $\widehat{\mathrm{AUC}}$ & $0.0003(0.0479)$ & $0.0013(0.0479)$ & $0.0055(0.0489)$ \\
\hline \multirow{6}{*}{$(80,80)$} & $\widehat{\alpha}_{1}$ & $0.162(1.3518)$ & $0.1531(1.3652)$ & 0.1594 (1.3561) \\
\hline & $\widehat{\alpha}_{2}$ & $0.2096(1.8697)$ & 0.2047 (3.9241) & $0.1655(2.183)$ \\
\hline & $\widehat{\beta}$ & $0.0372(0.4715)$ & $0.0438(0.5934)$ & $0.0458(0.7095)$ \\
\hline & $\hat{\lambda}_{1}$ & $0.0464(0.4011)$ & $0.0404(0.3996)$ & $0.0433(0.4048)$ \\
\hline & $\widehat{\lambda}_{2}$ & $0.0488(0.4211)$ & $0.0417(0.4334)$ & $0.0291(0.4084)$ \\
\hline & $\widehat{\mathrm{AUC}}$ & $0.002(0.0412)$ & $0.0023(0.0424)$ & $0.003(0.0435)$ \\
\hline
\end{tabular}

$$
\operatorname{RMSE}(\widehat{\mathrm{AUC}})=\sqrt{\frac{1}{1000} \sum_{i=1}^{1000}\left(\widehat{\mathrm{AUC}}_{i}-\mathrm{AUC}\right)^{2}}
$$

where $\widehat{\mathrm{AUC}}_{i}$ is the estimate of AUC for the $i$ th replicate.

Tables 1 and 2 report the ARB and the RMSE for the estimators of $\alpha_{1}, \alpha_{2}, \beta, \lambda_{1}, \lambda_{2}$, and the AUC. Table 1 reports the sensitivity analysis against $\alpha_{2}$ and sample sizes. In all cases, the two scale parameters have much smaller ARB and RMSE compared with shape parameters. The MLE of AUC behaves well. For two different shape parameters, it is always the common parameter $\beta$ which behaves better than the other two shape parameters $\alpha_{1}$ and $\alpha_{2}$ as expected. For two shape parameters $\alpha_{1}$ and $\alpha_{2}$, when $\alpha_{2}>\alpha_{1}, \alpha_{1}$ always behaves better than $\alpha_{2}$. Furthermore, as expected, the ARB and the RMSE decrease as the sample size increases. Table 2 reports the sensitivity analysis against the common shape parameter $\beta$. The results show the robustness of the MLEs for small sample sizes.

Our second simulations are to check the performance of MLE as the common shape parameter $\beta$ increases. We choose the following sample sizes: $(m, n)=(50,50),(80,80)$, $(50,80),(80,50)$. The two scale parameters are still set to 1 , and $\alpha_{1}=2, \alpha_{2}=3$ and $\beta$ takes three values: $2,2.5$ and 3 . The results are reported in Table 2 . Once again, one notices that AUC has very small ARB and RMSE. Under all settings, $\alpha_{2}$ has larger ARB and RMSE than that of $\alpha_{1}$ when $\alpha_{2}>\alpha_{1}$. When $\beta$ increases, we do not find a noticeable change of ARB and RMSE of all parameters.

\section{Conclusions}

In summary, this paper considers an Exponentiated Weibull model with two shape parameters and one scale parameter. Firstly, we derive a general moment formula which extends many results in the literature. Secondly, we obtain the theoretical AUC formula for a Bi-Exponentiated Weibull model which shares a common shape parameter between the two groups. We have noticed that the formula of AUC is independent of the common shape parameter, which is consistent with existing known results for simpler models such as Bi-generalized exponential and Bi-Weibull. Thirdly, we derive the maximum likelihood estimator of AUC for the $\mathrm{Bi}-\mathrm{EW}$ model with a common shape parameter and show the asymptotic normality of the estimators. Finally, we conduct a simulation study to illustrate the performance of our estimator for small to moderate sample sizes. The simulation results show that the MLE of AUC can behave very well under moderate sample sizes although the performance of the MLE of some shape parameters is somewhat unsatisfactory but still acceptable. 


\section{References}

[1] J. A. Hanley, "Receiver operating characteristic (ROC) methodology: the state of the art," Critical Reviews in Diagnostic Imaging, vol. 29, no. 3, pp. 307-335, 1989.

[2] C. B. Begg, "Advances in statistical methodology for diagnostic medicine in the 1980's," Statistics in Medicine, vol. 10, no. 12, pp. 1887-1895, 1991.

[3] M. S. Pepe, The Statistical Evaluation of Medical Tests for Classification and Prediction, vol. 28 of Oxford Statistical Science Series, Oxford University Press, Oxford, 2003.

[4] D. M. Green and J. A. Swets, Singal Detection Theory and Psychophysics, Wiley and Sons, New York, NY, USA, 1966.

[5] H. Fushing and B. W. Turnbull, "Nonparametric and semiparametric estimation of the receiver operating characteristic curve," The Annals of Statistics, vol. 24, no. 1, pp. 25-40, 1996.

[6] C. E. Metz, B. A. Herman, and J. H. Shen, "Maximum likelihood estimation of receiver operating characteristic curve from continuous distributed data," Statistics in Medicine, vol. 17, pp. 1033-1053, 1998.

[7] K. H. Zou and W. J. Hall, "Two transformation models for estimating an ROC curve derived from continuous data," Journal of Applied Statistics, vol. 5, pp. 621-631, 2000.

[8] J. Gu, S. Ghosal, and A. Roy, Non-Parametric Estimation of ROC Curve, Institute of Statistics Mimeo Series, 2005.

[9] M. S. Pepe and T. Cai, "The analysis of placement values for evaluating discriminatory measures," Biometrics, vol. 60, no. 2, pp. 528-535, 2004.

[10] X.-H. Zhou and H. Lin, "Semi-parametric maximum likelihood estimates for ROC curves of continuous-scales tests," Statistics in Medicine, vol. 27, no. 25, pp. 5271-5290, 2008.

[11] D. Faraggi, B. Reiser, and E. F. Schisterman, "ROC curve analysis for biomarkers based on pooled assessments," Statistics in Medicine, vol. 22, no. 15, pp. 2515-2527, 2003.

[12] A. J. Simpson and M. J. Fitter, "What is the best index of detectability?” Psychological Bulletin, vol. 80, no. 6, pp. 481-488, 1973.

[13] M. H. Gail and S. B. Green, "A generalization of the onesided two-sample Kolmogorov-Smirnov statistic for evaluating diagnostic tests," Biometrics, vol. 32, no. 3, pp. 561-570, 1976.

[14] W.-C. Lee and C. K. Hsiao, "Alternative summary indices for the receiver operating characteristic curve," Epidemiology, vol. 7, no. 6, pp. 605-611, 1996.

[15] G. Campbell, "Advances in statistical methodology for the evaluation of diagnostic and laboratory tests," Statistics in Medicine, vol. 13, no. 5-7, pp. 499-508, 1994.

[16] S. Kotz, Y. Lumelskii, and M. Pensky, The Stress-Strength Model and Its Generalizations, 2002.

[17] A. M. Awad and M. A. Hamadan, "Some inference results in $\operatorname{Pr}(X<Y)$ in the bivariate exponential model," Communications in Statistics, vol. 10, no. 24, pp. 2515-2524, 1981.

[18] W. A. Woodward and G. D. Kelley, "Minimum variance unbiased estimation of $P(Y<X)$ in the normal case," Technometrics, vol. 19, pp. 95-98, 1997.

[19] K. Constantine and M. Karson, "The Estimation of $P(X>Y)$ in Gamma case," Communication in Statistics-Computations and Simulations, vol. 15, pp. 65-388, 1986.

[20] K. E. Ahmad, M. E. Fakhry, and Z. F. Jaheen, "Empirical Bayes estimation of $P(Y<X)$ and characterizations of Burr-type $X$ model," Journal of Statistical Planning and Inference, vol. 64, no. 2, pp. 297-308, 1997.
[21] J. G. Surles and W. J. Padgett, "Inference for $P(X>Y)$ in the Burr type X model," Journal of Applied Statistical Sciences, vol. 7, pp. 225-238, 1998.

[22] J. G. Surles and W. J. Padgett, "Inference for reliability and stressstrength for a scaled Burr type X distribution," Lifetime Data Analysis, vol. 7, no. 2, pp. 187-200, 2001.

[23] M. Z. Raqab and D. Kundu, "Comparison of different estimation of $P(X>Y)$ for a Scaled Burr Type X Distribution," Communications in Statistica-Simulation and Computation, vol. 22, pp. 122-150, 2005.

[24] D. Kundu and R. D. Gupta, "Estimation of $P(X>Y)$ for generalized exponential distribution," Metrika, vol. 61, no. 3, pp. 291-308, 2005.

[25] D. Kundu and R. D. Gupta, "Estimation of $P(X>Y)$ For Weibull distribution," IEEE Transactions on Reliability, vol. 34, pp. 201226, 2006.

[26] E. W. Stacy, "A generalization of the gamma distribution," Annals of Mathematical Statistics, vol. 33, pp. 1187-1192, 1962.

[27] R. L. Prentice, "Discrimination among some parametric models," Biometrika, vol. 62, no. 3, pp. 607-614, 1975.

[28] D. J. Slymen and P. A. Lachenbruch, "Survival distributions arising from two families and generated by transformations," Communications in Statistics A, vol. 13, no. 10, pp. 1179-1201, 1984.

[29] D. P. Gaver and M. Acar, "Analytical hazard representations for use in reliability, mortality, and simulation studies," Communications in Statistics B, vol. 8, no. 2, pp. 91-111, 1979.

[30] U. Hjorth, "A reliability distribution with increasing, decreasing, constant and bathtub-shaped failure rates," Technometrics, vol. 22, no. 1, pp. 99-107, 1980.

[31] G. S. Mudholkar and D. K. Srivasta, "Exponentiated weibull family: a reanalysis of the bus-motor-failure data," Technometrics, vol. 37, no. 4, pp. 436-445, 1995.

[32] G. S. Mudholkar and A. D. Hutson, "The exponentiated Weibull family: some properties and a flood data application," Communications in Statistics, vol. 25, no. 12, pp. 3059-3083, 1996.

[33] A. L. Khedhairi, A. Sarhan, and L. Tadj, Estimation of the Generalized Rayleigh Distribution Parameters, King Saud University, 2007.

[34] A. Baklizi, "Inference on $\operatorname{Pr}(X<Y)$ in the two-parameter Weibull model based on records," ISRN Probability and Statistics, vol. 2012, Article ID 263612, 11 pages, 2012.

[35] L. Qian, "The Fisher information matrix for a three-parameter exponentiated Weibull distribution under type II censoring," Statistical Methodology, vol. 9, no. 3, pp. 320-329, 2012.

[36] A. Choudhury, "A simple derivation of moments of the exponentiated Weibull distribution," Metrika, vol. 62, no. 1, pp. 17-22, 2005. 


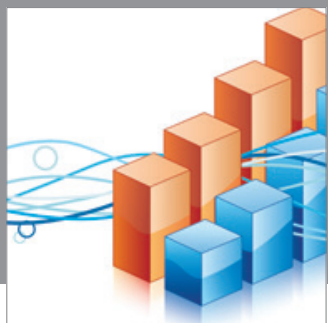

Advances in

Operations Research

mansans

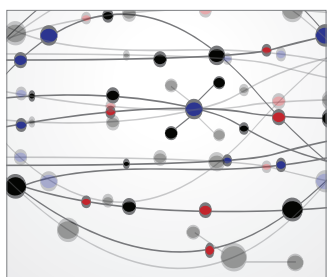

The Scientific World Journal
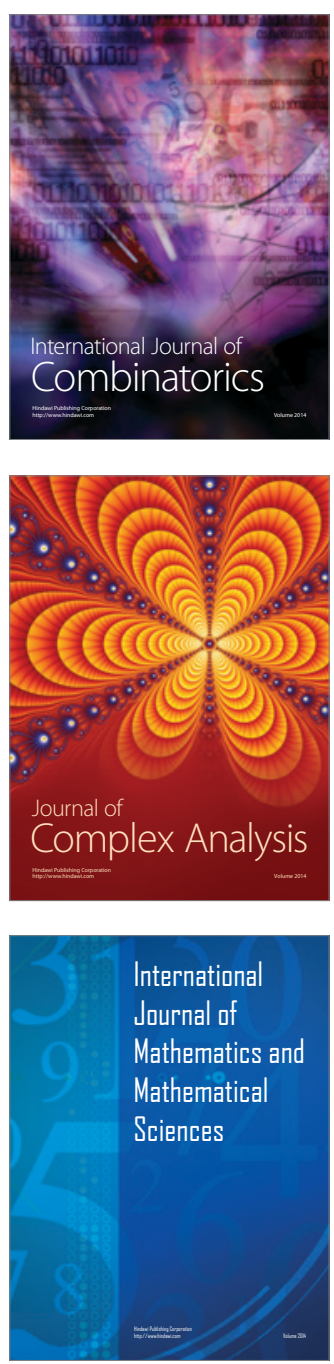
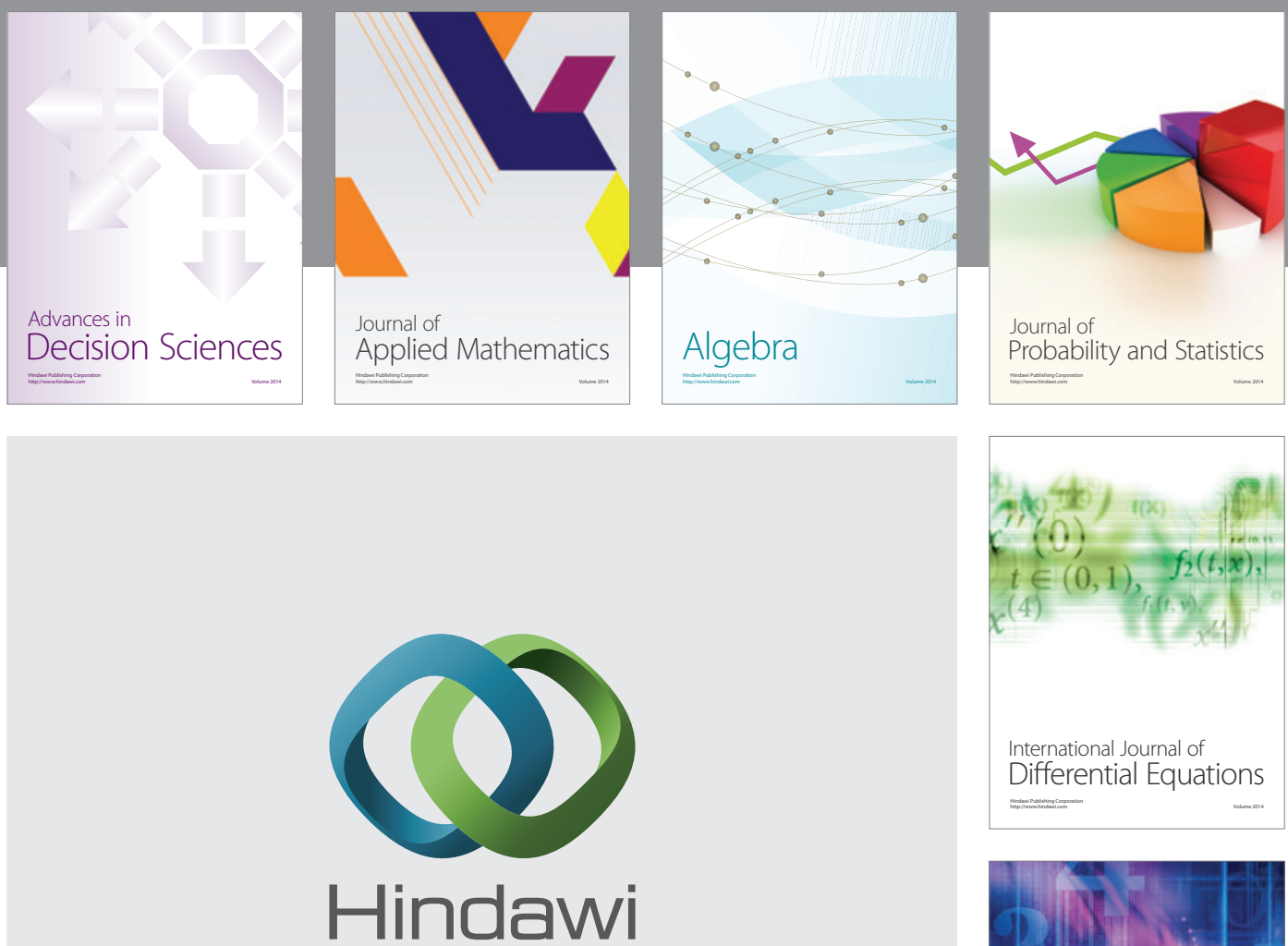

Submit your manuscripts at http://www.hindawi.com
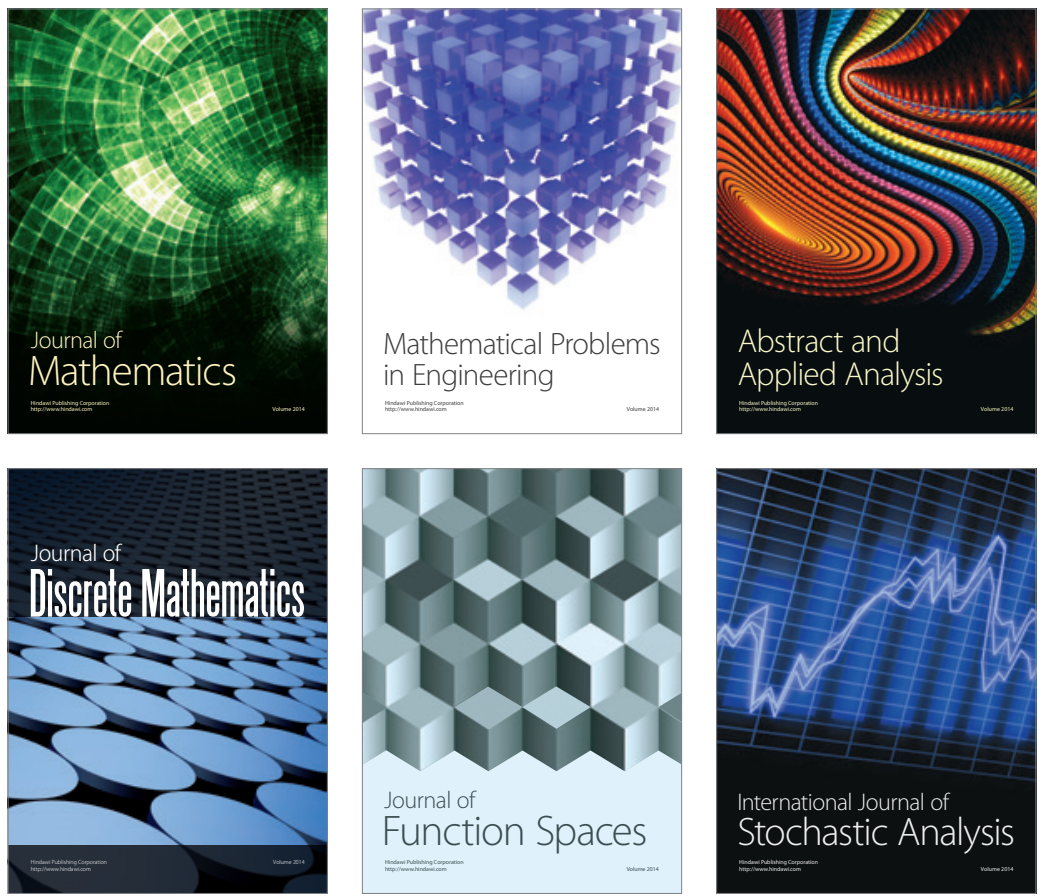

Journal of

Function Spaces

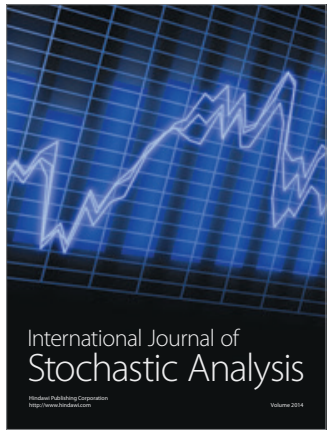

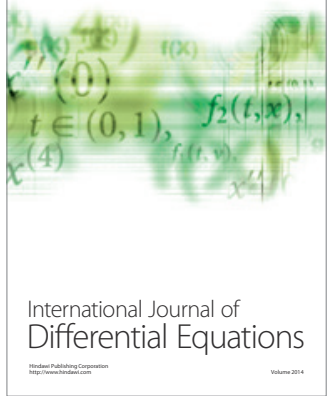
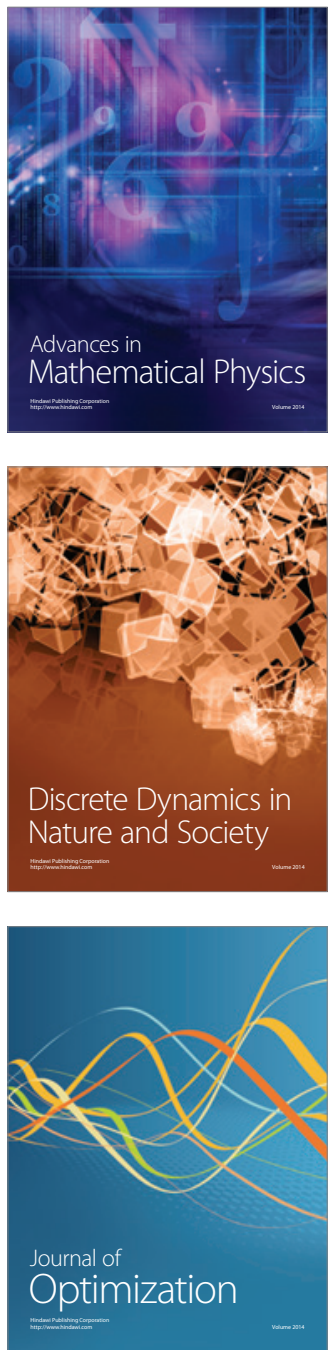